\title{
The Effect of Resistance and Balance Training on Postural Control and Physical Fitness in Adults with Down Syndrome
}

\author{
Yun-A Shin $\mathrm{PhD}^{1}$, Sang-Min Hong $\mathrm{PhD}^{2}$, Jong-Sun Lee $\mathrm{PhD}^{3}$, Hyo-Been Jeong MS ${ }^{4}$ \\ 1Department of Exercise Prescription \& Rehabilitation, Dankook University, Cheonan; ${ }^{2}$ Department of Sports Rehabilitation, Gimcheon University, Gimcheon; ${ }^{3}$ Ong-I Jin-I \\ Sports Promotion Agency, Incheon; ${ }^{4}$ Graduate School of Physical Education, Dongguk University, Seoul, Korea
}

PURPOSE: This study aimed to examine the effects of resistance and balance training on physical function and postural control in individuals with Down syndrome (DS).

METHODS: Ten adults with DS constituted the experimental group (EX) and attended an 8-week resistance and balance training program. The results were compared with those of the control group (CON), which consisted of 10 patients who did not undergo any physical training. Body composition, postural stability, and physical fitness were measured before and after the training program. Resistance and balance training were performed for 60 minutes, three times a week for 8 weeks.

RESULTS: Body weight, body mass index, body fat percentage, and waist circumference decreased significantly in the EX group after completing the 8-week resistance and balance training program. The center of pressure and the difference between standing foot pressure on the left and right were significantly improved in the EX group. Physical fitness including sit-to-stand repetitions and $10 \mathrm{~m}$ shuttle duration significantly improved in the EX and CON groups.

CONCLUSIONS: These results suggest that resistance and balance training to improve musculoskeletal problems is an effective strategy to prevent injury, fatigue, and falls during exercise and to improve general health in individuals with DS.

Key words: Down syndrome, Resistance training, Postural balance, Physical fitness

\section{INTRODUCTION}

As individuals age, physical function plays a vital role in health and independence [1]. Low physical function was associated with musculoskeletal health conditions, such as loss of muscle mass and an increased risk of falls [2]. Although, the aging population with Down syndrome (DS) is increasing as a result of improved healthcare services provided in the community in the past seven decades [3,4], the ageing population in this group is at an increased risk of deconditioning and morbidity induced by DS [5]. The presence of an additional third chromosome 21 or trisomy 21 caused by a chromosomal disorder due to an error in cell division, as is characteristic of DS, results in precocious ageing within this group [6].

Moreover, numerous studies have reported that individuals with DS are inherently prone to joint laxity, muscle hypotonia, low levels of muscle strength, and deficits of the cerebellum [6,7]. Muscle hypotonia is suggested to be directly related to impaired postural control and gait alterations $[8,9]$. Motor disability is most common in individuals with DS and it includes longer motion and reaction times, stability and postural deficits $[10,11]$. These limitations in physical function lead to neuromuscular disorders, such as hallux valgus, hammer toe deformities, plantar fasciitis, and flat feet, which impair ambulation and cause further dysfunction [6].

In particular, people with an intellectual disability have a rapid initial

Corresponding author: Sang-Min Hong Tel +82-10-2421-9933 Fax +82-54-420-4434 E-mail rippey5070/anaver.com

*This study was supported by the Ministry of Education of the Republic of Korea and the National Research Foundation of Korea (NRF-2017S1A5A8020453). Keywords Down syndrome, Resistance training, Postural balance, Physical fitness

Received 1 Mar 2021 Revised 26 Apr 2021 Accepted 27 Apr 2021 
decrease in muscle mass which is a symptom associated with premature aging, and this symptom indicates a rate of functional decline with respect to lower limb muscle strength in the early forties [12-15]. Interventions that prevent low physical function are the most important to maintain activities of daily living (ADL), prevent the need for care dependency and increased healthcare costs [16-18]. Therefore, adequate exercise training, which is considered appropriate for muscular disorders, is needed to maintain and enhance physical function in older individuals with DS.

In general, resistance exercises are known to be more effective in increasing muscle mass than aerobic exercises [19], however, most studies have reported the effects of aerobic exercise $[20,21]$ and combined exercise [22,23]. Carmeli et al. [24] reported that low muscle mass and strength have a role to play in the early onset of functional deterioration in older individuals with intellectual disability. However, only a few studies $[25,26]$ have examined the effects of resistance training in individuals with DS, especially in adults or the elderly with DS.

Additional study on maintaing the physical ability and managing health of in adults or the elderly with DS are needed. Moreover, DS has a short life span and deteriorated with age, therefore In particular, there are few studies on individuals with DS residing in nursing homes that require a lot of care.

Therefore, we examined the effect of resistance and balance training exercises that were considered appropriate for muscular disorders, in the prevention of muscular disease. Their effectiveness in enhancing muscular strength for postural control and preventing falls in individuals with DS was also evaluated.

\section{METHODS}

\section{Subjects}

The subjects in this study consisted of 20 people (female, $n=5$; male, $\mathrm{n}=15$ ) with intellectual disabilities aged between 30 to 50 years old in I city (mean age: $44.55 \pm 7.25$ years). All the participants were residents of a nursing home in I city and informed about the experiment. The only subjects selected were those with intellectual disabilities due to DS. These participants did not exercise regulary within 6 moths. Additional inclusion criteria include: 1) Functional ability sufficient to understand conversation, exercise guidance, and direction, 2) Reside in a supported living condition have a caregiver. Participants with intellectual disabilities, such as those caused by encephalitis, meningitis, disorders due to metabolic disorders, vitamin deficiency, and tumors were excluded from the study. All the participants were independent ambulators. The participants provided informed consent to participate in the study and this study was approved by the Ethical Committee of the Dongguk University institutional review board community (DUIRB-201708-01).

\section{Physical examination}

Anthropometric measurements were performed by trained and certified observers. Height, body weight, and body fat percent were recorded (INBODY 270, InBody, Korea). Body mass index (BMI) was calculated using the ratio of weight $(\mathrm{kg}) /$ height $\left(\mathrm{m}^{2}\right)$. Waist circumference was determined at the level of the natural waist between the ribs and the iliac crest at the end of a normal expiration.

\section{Physical fitness}

After a brief warm-up, participants from the experimental (EX) and control groups $(\mathrm{CON})$ were measured for their physical fitness. The following measurements were included:

\section{1) Sit-to-stand test}

This tests muscle endurance and is a measure of the time taken to complete a full stand from a seated position. During the test, subjects were required to fold their arms across their chest in order to use as much of their lower muscle strength as possible. The reliability of this test for adults with intellectual disabilities was reported to be 0.72 [27].

\section{2) Grip strength}

To measure muscle strength. Subjects were required to stand with their legs at shoulder width apart and lower their arms to the side. The grip was adjusted to match the second bar of your finger. The participants were then instructed to hold the dynamometer with one hand and grip it as hard as they could. The right and left sides were examined twice and the best values were recorded. The reliability of this test for adults with mental disabilities was reported to be 0.94 [27].

\section{3) Incremental shuttle walking test (ISWT) for $10 \mathrm{~m}$}

It is a test in which a participant walks a distance of $10 \mathrm{~m}$ with the inspector at a speed of $0.50 \mathrm{~m} / \mathrm{s}$. Every minute, the inspector increases the speed by $0.17 \mathrm{~m} / \mathrm{s}$ according to the inspection method. The time is estimated by recording the distance walked at a given speed, allowing the participant to maintain their speed [28]. 


\section{4) The $5 \mathrm{~m}$ walking test}

This is an estimate of the force of movement and a combination of strength and dynamic balance. This test measures the time taken to walk a $5 \mathrm{~m}$ straight distance on a flat ground with the usual gait, paying attention not to run. The reliability of the test for the mentally disabled was reported to be 0.96 .

\section{Static postural control examination}

This method measures the ability to control a standing posture. The subject stands on the balance force platform (Zebris PDM-system, Zebris Medical GmbH, Germany) for 1 minute with their feet shoulder width apart. They were asked to maintain their standing position with open eyes focusing on a $6 \mathrm{~m}$ black circle $1.5 \mathrm{~m}$ away in their vision line [29]. The degree of sway in millimeters while standing and the difference between the weight load on the left and right were measured.

\section{Exercise program}

The exercise program was managed and assisted by a team of five exercise specialists. Three special sports specialists and two exercise specialists instructed the strength and balance exercise program. In the morning and afternoon, 10 DS patients participated in the exercise program. Each class consisted of five people and there were four exercise program classes, from 09:00 am to 10:00 am, 10:30 am to 11:30 am, 14:00 to $15: 00 \mathrm{pm}$, and 15:30 to $16: 30 \mathrm{pm}$. Every session was composed of 60 minutes including a 10-minute warm-up, followed by 40 minutes of resistance training, and a 10-minute cool-down. All movements were repeated 15 times during a given time and three sets were performed. Exercise intensity was used as rating of perceived exertion (RPE) and was set at moderated intensity (12-13 level). This was done 3 times per week for 8 weeks. In order to improve abnormal postural stability and walking patterns in patients with DS, exercises that can improve the deformation of hip and knee joint were required. Since resistance and stretching exercises have been suggested for improving postural stability and walking ability, the exercise program in this study consisted of exercises that are known to strengthen the muscles around the hip joint and ankle joint, and it included stretching for the lower back muscles. In particular, a muscle strengthening program of the gluteus maximus, gluteus medius, quadriceps femoris, adductor magnus, tibialis posterior, ankle flexor and extensor muscle, and a stretching program of the iliopsoas and biceps femoris were the focus areas in this study. Additionally, it is important to note that careful attention should be given to stretching the muscles of a patient with DS, because they are inherently loose in the joints and ligaments. Therefore, the stretching program consisted of dynamic movements because light dynamic stretching is preferred over static stretching to prevent injury during exercise [30]. Taking these factors into consideration, the exercise program used in this study is shown in Table 1.

\section{Statistical analysis}

Results were expressed as mean \pm standard deviation using the SPSS/ PC statistic program (version 20.0 for windows; SPSS, Inc., Chicago, IL, USA). Independent t-test was used to determine the significant differences of comparisons between the groups at baseline. The changes in body composition, postural control, and physical fitness pre and post the resistance and balance exercise program were assessed by Two-way repeated analysis of variance. Differences were considered statistically significant if $\alpha=.05$.

\section{RESULTS}

After the 8-week resistance and balance training program, the changes in body composition are shown in Table 2. Body weight $(p<.05)$, BMI $(p<.05)$, body fat percent $(p<.05)$ and waist circumference $(p<.001)$ showed the effect of interaction between time and group except fat-free mass.

The changes in center of pressure (COP) and standing foot pressure difference after 8 weeks of the resistance and balance training program are shown in Table 3. COP showed a significant difference after the exercise training program $(p<.01)$ and the effect of interaction between time and group $(p<.001)$. Weight load difference showed a significant difference after the training program $(p<.05)$, between groups $(p<.01)$, and the effect of interaction between time and group $(p<.05)$.

The changes in fitness factors after 8 weeks of the strength and balance program are shown in Table 4 . The number of sit-to-stand repetitions showed a significant difference after the exercise program $(p<.05)$, and the number of repetitions in both groups increased significantly. The $10 \mathrm{~m}$ shuttle time also showed a significant difference after the exercise program $(p<.01)$, and the EX group showed a significantly improved $10 \mathrm{~m}$ shuttle time. 
Table 1. The combined 8-week exercise program for individuals with Down syndrome

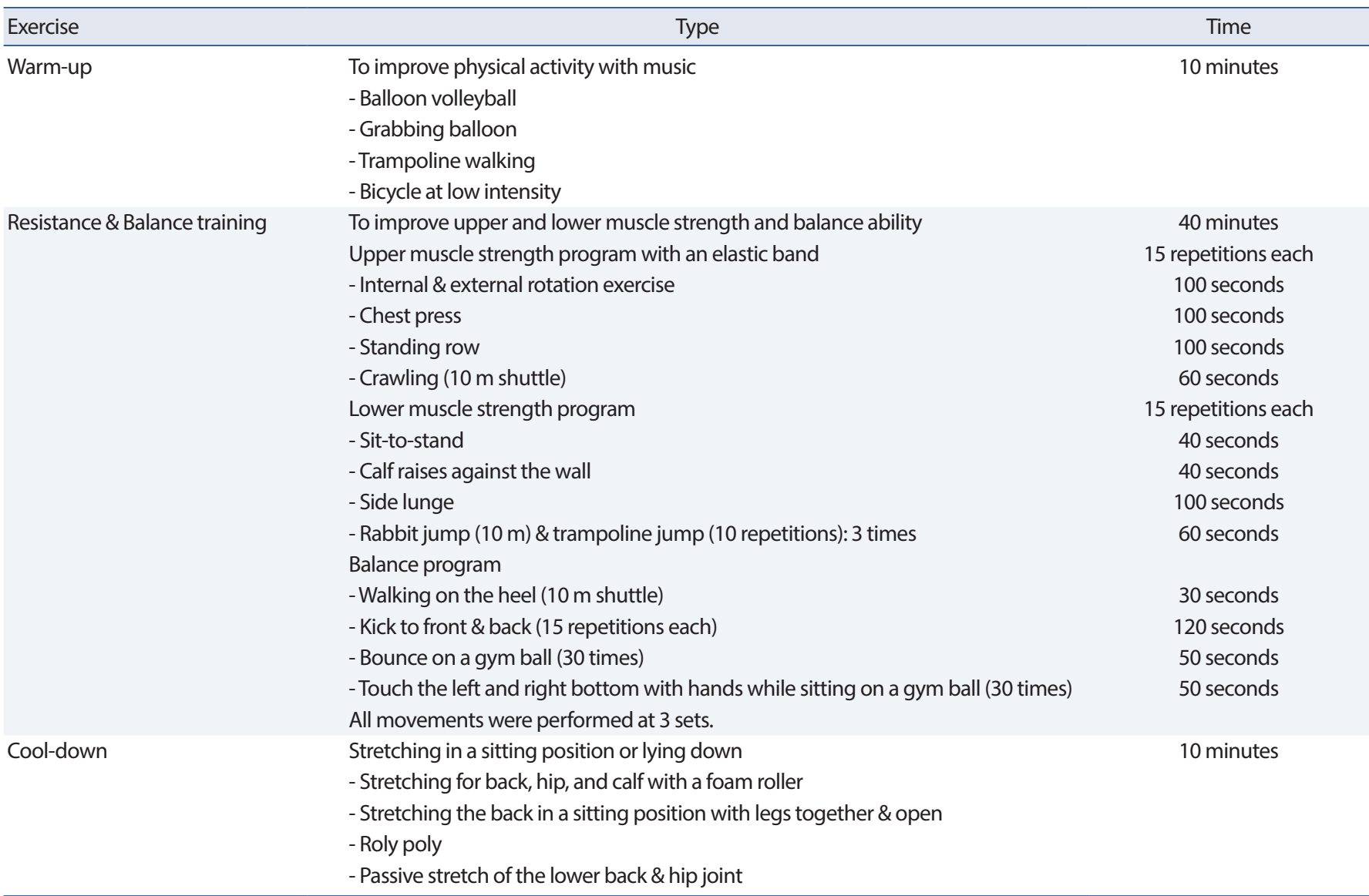

Table 2. Changes in body composition after the resistance and balance training program

\begin{tabular}{|c|c|c|c|c|c|c|}
\hline Variables & Group & Pre & Post & & & Sig. \\
\hline \multirow[t]{3}{*}{ Weight (kg) } & EX & $64.52 \pm 12.81$ & $62.88 \pm 12.47$ & $\mathrm{~T}$ & 2.743 & .120 \\
\hline & CON & $69.90 \pm 7.70$ & $70.32 \pm 8.41$ & $\mathrm{G}$ & 1.31 & .263 \\
\hline & & & & $T^{*} G$ & 8.033 & $.013^{*}$ \\
\hline \multirow[t]{3}{*}{ BMI $\left(\mathrm{kg} / \mathrm{m}^{2}\right)$} & EX & $25.18 \pm 5.51$ & $24.54 \pm 5.53$ & $\mathrm{~T}$ & 3.612 & .078 \\
\hline & $\mathrm{CON}$ & $26.48 \pm 2.65$ & $26.62 \pm 2.71$ & $\mathrm{G}$ & .552 & .470 \\
\hline & & & & $T^{*} G$ & 8.966 & $.010^{*}$ \\
\hline \multirow[t]{3}{*}{ FFM (kg) } & EX & $25.88 \pm 4.48$ & $26.63 \pm 4.99$ & $\mathrm{~T}$ & .438 & .518 \\
\hline & $\mathrm{CON}$ & $25.14 \pm 4.85$ & $24.80 \pm 4.61$ & $\mathrm{G}$ & .313 & .584 \\
\hline & & & & $T^{*} G$ & 3.192 & .094 \\
\hline \multirow[t]{3}{*}{$\%$ FAT (\%) } & EX & $31.08 \pm 5.75$ & $28.43 \pm 6.74$ & $\mathrm{~T}$ & 1.924 & .186 \\
\hline & $\mathrm{CON}$ & $29.61 \pm 7.17$ & $30.19 \pm 6.75$ & $\mathrm{G}$ & .002 & .963 \\
\hline & & & & $T^{*} G$ & 4.667 & $.047^{*}$ \\
\hline \multirow[t]{3}{*}{ Waist (cm) } & EX & $87.0 \pm 11.27$ & $84.15 \pm 10.52$ & $\mathrm{~T}$ & 2.401 & .142 \\
\hline & CON & $92.0 \pm 6.50$ & $93.0 \pm 7.63$ & $\mathrm{G}$ & 2.193 & .159 \\
\hline & & & & $T^{*} G$ & 10.401 & $.006^{* *}$ \\
\hline
\end{tabular}

T, time; G, group; $T^{*} G$, the interaction between time and group; EX, exercise group; CON, control group; BMl: body mass index; FFM, fat-free mass; \%FAT: body fat percent.

\section{DISCUSSION}

The exercises used in this study consisted of strength and balance training to improve musculoskeletal problems for upper and lower extremity strength and postural balance in patients with DS. After 8 weeks of strength and balance training in this study, body composition, bal- 
Table 3. Changes in COP and standing foot pressure after the strength and balance training program

\begin{tabular}{lcccrrr}
\hline Variables & Group & Pre & Post & & F & Sig. \\
\hline COP $(\mathrm{mm})$ & EX & $1,495.08 \pm 910.09$ & $145.71 \pm 58.81$ & T & 25.039 & .000 \\
& CON & $1,466.17 \pm 1,096.89$ & $1,418.37 \pm 931.08$ & G & 1.798 & .200 \\
Weight load $(\mathrm{kPa})$ & & & & $T^{*} \mathrm{G}$ & 11.931 & .004 \\
& EX & $31.96 \pm 18.34$ & $13.94 \pm 4.76$ & $\mathrm{~T}$ & 8.295 & .011 \\
& CON & $47.13 \pm 18.76$ & $45.58 \pm 17.09$ & $\mathrm{G}$ & 11.239 & .004 \\
& & & & $T^{*} \mathrm{G}$ & 5.868 & .029 \\
\hline
\end{tabular}

EX, exercise group; CON, control group; COP, center of pressure.

Table 4. Changes in physical fitness after 8 weeks of the strength and balance training program

\begin{tabular}{|c|c|c|c|c|c|c|}
\hline Variables & Group & Pre & Post & & & Sig. \\
\hline \multirow[t]{3}{*}{ Sit-to-Stand (number) } & EX & $9.55 \pm 4.29$ & $13.36 \pm 3.88$ & $\mathrm{~T}$ & 6.599 & .021 \\
\hline & CON & $10.71 \pm 3.25$ & $11.43 \pm 2.50$ & $\mathrm{G}$ & .061 & .808 \\
\hline & & & & $T^{*} G$ & 3.095 & .098 \\
\hline \multirow[t]{3}{*}{ Muscle Strength (kg) } & EX & $14.58 \pm 7.73$ & $16.84 \pm 6.55$ & $\mathrm{~T}$ & 1.367 & .258 \\
\hline & CON & $16.82 \pm 7.61$ & $16.42 \pm 7.31$ & $\mathrm{G}$ & .076 & .785 \\
\hline & & & & $T^{*} G$ & 2.793 & .113 \\
\hline \multirow[t]{3}{*}{ ISWT (sec) } & EX & $125.09 \pm 33.49$ & $195.86 \pm 95.18$ & $\mathrm{~T}$ & 10.399 & .005 \\
\hline & CON & $107.43 \pm 36.85$ & $151.09 \pm 34.44$ & G & .592 & .453 \\
\hline & & & & $T^{*} \mathrm{G}$ & 3.095 & .098 \\
\hline \multirow[t]{3}{*}{$5 \mathrm{~m}$ walking (sec) } & EX & $5.36 \pm 1.40$ & $5.02 \pm 1.06$ & $\mathrm{~T}$ & 0.452 & .511 \\
\hline & CON & $5.82 \pm .70$ & $5.68 \pm 1.82$ & G & 1.175 & .294 \\
\hline & & & & $T^{*} \mathrm{G}$ & .074 & .789 \\
\hline
\end{tabular}

EX, exercise group; CON, control group; ISWT, 10 m incremental shuttle walking test.

ance ability, and physical fitness factors such as sit-to-stand repetitions and ISWT walking time improved significantly.

Long-term exercise has been reported to reduce body fat and increase muscle mass in a study of people with intellectual disabilities [20]. However, in a study where aerobic and resistance exercises were performed separately by patients with DS, aerobic exercises decreased body weight and body fat percent [20,21], however, resistance exercises have shown inconsistent results $[23,31]$. In this study, resistance and balance exercises for 8 weeks significantly decreased body weight, BMI, and body fat percentage. Moreover, fat free mass increased in the EX group, but this result was insignificant. Insignificant changes in fat free mass were probably due to a relatively short period of the exercise intervention (8 weeks), and it is plausible that a longer period of intervention might result in a greater improvement in fat free mass.

The result of the changes in body composition after a relatively shortterm resistance training program was significant. An increase in BMI is related to an increase in functional impairment [32], which may induce an impaired balance and increase the risk of falling thereby causing postural stress and perturbation [33,34], even in individuals aged under 40 years $[35,36]$. However, weight reduction has a favorable impact on pos- tural instability [36]. Participants with DS in this study showed a higher BMI and body fat percent before the exercise program. Therefore, when considered together, a high body weight and the physical characteristics of DS, can impact postural balance negatively.

Furthermore, DS was reported to reduce an individual's capacity to maintain postural stability, because postural stability requires the simultaneous combination of motion and stability of the ankle and hip [37]. It has been reported that a decrease in muscle hypotonia induces muscle weakness, more flexibility in the joint than necessary, and low balance ability in patients with DS. In this study, patients with DS showed a higher frequency of postural sway and a greater difference between weight load on the left and right in the standing position before the exercise program. However, the EX group had reduced postural sway and a lower difference between weight load on the left and right in the standing position after the 8-week exercise program. A previous study recommended that strengthening the ankle flexors and extensors and balance training to improve medial-lateral control using hip strategies should be considered in rehabilitation programs [38]. As our training program in this study focused on the ankle and hip joint muscle group, this program could have a favorable impact on postural balance as well as body 
weight reduction.

In this study, the number of sit-to-stand repetitions and the $10 \mathrm{~m}$ shuttle time increased after 8 weeks. Skeletal deformation of the foot in DS causes extensive fatigue in the calf and foot after repeated walking and weakening of the tibialis posterior, which assists the longitudinal arch [39]. Passive and active massage or stretching and tibialis posterior strengthening exercises are required to improve calf muscle and plantar fatigue. Additional exercises were recommended as balance exercises that were closely related to one's ability to walk, for example, standing up against the wall with raised toes, standing in place with the heels up, and walking on the heel [37]. Our training focused and consisted of a program based on these strategies, therefore, the participants with DS in the EX group in this study showed an improvement in lower muscle endurance and the $10 \mathrm{~m}$ shuttle duration.

Previous studies of resistance training alone reported significantly improved maximal muscle strength after upper and lower muscle exercises 3 times per week $[25,40]$, however, resistance training alone was shown to have limited improvements in maximal muscle strength after upper and lower muscle exercises twice per week [26]. However, it has been reported that muscle strength improved after aerobic exercises that targeted a large muscle group of the body were performed twice a week, and previous studies suggested that combined exercises were more effective than resistance exercises alone. In other words, combined exercises showed improvement in cardiopulmonary function as well as muscle strength and these results suggest that combined exercises are more efficient than aerobic or strength exercises alone.

However, inherit musculoskeletal dysfunction in DS causes secondary muscle loss and body deformation, resulting in various musculoskeletal disorders and joint instability [6]. Therefore, we suggested that strength training to improve these musculoskeletal problems should be performed at the beginning of an exercise class and then proceed to combined exercises. This strategy is expected to be more effective in preventing injury, fatigue, and falls that can occur during exercise and to improve the health in individuals with DS.

As a resutls of this study, it was found that balance, muscular endurance, and walking capacity were increased after 8 weeks. Our training program in this study focused on the ankle and hip joint muscle group, this program could have a favorable impact on postural balance as well as body weight reduction. Our training also focused and consisted of a program based on related to one's ability to walk balance. Therefore, training program of this study that increase muscle strength related to balance was effective in improving muscular strength for postural control and preventing falls in individuals with DS.

\section{CONCLUSION}

This study was conducted on individuals with DS living in the same area, the diet and lifestyle habits had the similar patterns, because participants were a residents in nursing home. Therefore, exogenous variable that may affect the the effect of exercise were minimized. However, as a limitation of our study, the number of subjects were only 20 , and the study period was only 8 weeks. Further study are needed to expand to larger study population and apply a longer period of exercise duration.

\section{CONFLICT OF INTEREST}

No potential conflict of interest relevant to this article was reported.

\section{AUTHOR CONTRIBUTIONS}

Conceptualization: YA Shin; Data curation: JS Lee; Formal analysis: HB Jeong; Funding acquisition: SM Hong; Methodology: YA Shin; Project administration: SM Hong; Visualization: JS Lee; Writing-original draft: YA Shin; Writing-review \& editing: SM Hong.

\section{ORCID}

$\begin{array}{ll}\text { Yun-A Shin } & \text { https://orcid.org/0000-0002-8480-3454 } \\ \text { Sang-Min Hong } & \text { https://orcid.org/0000-0003-4462-9217 } \\ \text { Jong-Sun Lee } & \text { https://orcid.org/0000-0002-0273-9445 } \\ \text { Hyo-Been Jeong } & \text { https://orcid.org/0000-0002-7218-9477 }\end{array}$

\section{REFERENCES}

1. Tudor-Locke C, Washington TL, Hart TL. Expected values for steps/ day in special populations. Prev Med. 2009;49(1):3-11.

2. Mazzeo RS, Tanaka H. Exercise prescription for the elderly: current recommendations. Sports Med. 2001;31(11):809-18.

3. Patja K, Mölsä P, Iivanainen M. Cause-specific mortality of people with intellectual disability in a population-based, 35-year follow-up study. J Intellect Disabil Res. 2001;45(1):30-40. 
4. Rimmer JH, Braddock D. Health promotion for people with physical, cognitive, and sensory disabilities: an emerging national priority. Am J Health Promot. 2002;16(4):220-4.

5. Ashman AF, Suttie JN. The social and community involvement of older australians with intellectual disabilities. J Intellect Disabil Res. 1996;40(2):120-9.

6. Roizen NJ, Patterson D. Down's syndrome. Lancet. 2003;361(9365): 1281-9.

7. Jensen KM, Taylor LC, Davis MM. Primary care for with Down syndrome: adherence to preventive healthcare recommendations. J Intellect Disabil Res. 2013;57(5):409-21.

8. Cowie VA. A study of the early development of Mongols. Oxford, UK: Pegramon Press 1970.

9. Davis WE, Kelso JA. Analysis of "invariant characteristics" in the motor control of Down's syndrome and normal subjects. J Mot Behav. 1982;14(3):194-212.

10. Aruin AS, Almeida GL, Latash ML. Organization of a simple twojoint synergy in individuals with down syndrome. AM J Ment Retard. 1996;101(3):256-68.

11. Shumway-Cook A, Woollacott MH. Dynamics of postural control in the child with down syndrome dynamics of postural control in the child with down syndrome. Phys Ther. 1985;65(9):1315-22.

12. Batty GD, Deary IJ, Macintyre S. Childhood IQ in relation to risk factors for premature mortality in middle-aged persons: the aberdeen children of the 1950s study. J Epidemiol Community Health. 2007;61 (3):241-7.

13. Janicki MP, Dalton AJ, Henderson CM, Davidson PW. Mortality and morbidity among older adults with intellectual disability: health services considerations. Disabil Rehabil. 1999;21(5-6):284-94.

14. Kilgour AH, Starr JM, Whalley LJ. Associations between childhood intelligence (IQ), adult morbidity and mortality. Maturitas. 2010;65 (2):98-105.

15. Torr J, Davis R. Ageing and mental health problems in people with intellectual disability. Curr Opin Psychiatry. 2007;20(5):467-71.

16. Bartlo P, Klein PJ. Physical activity benefits and needs in adults with intellectual disabilities: systematic review of the literature. Am J Intellect Dev Disabil. 2011;116(3):220-32.

17. Calders P, Elmahgoub S, Roman de Mettelinge T, Vandenbroeck C, Dewandele I, et al. Effect of combined exercise training on physical and metabolic fitness in adults with intellectual disability: a controlled trial. Clin Rehabil. 2011;25(12):1097-108.
18. Heller T, McCubbin JA, Drum C, Peterson J. Physical activity and nutrition health promotion interventions: what is working for people with intellectual disabilities? Intellect Dev Disabil. 2011;49(1):26-36.

19. Donges CE, Duffield R, Drinkwater EJ. Effects of resistance or aerobic exercise training on interleukin-6, c-reactive protein, and body composition. Med Sci Sports Exerc. 2010;42:304-13.

20. Mendonca GV, Pereira FD. Influence of long-term exercise training on submaximal and peak aerobic capacity and locomotor economy in adult males with Down's syndrome. Med Sci Monit. 2009;15(2):CR33-9.

21. Ordonez FJ, Rosety M, Rosety-Rodriguez M. Influence of 12-week exercise training on fat mass percentage in adolescents with down syndrome. Med Sci Monit. 2006;12(10):416-9.

22. Elmahgoub SM, Lambers S, Stegen S, Van Laethem C, Cambier D, et al. The influence of combined exercise training on indices of obesity, physical fitness and lipid profile in overweight and obese adolescents with mental retardation. Eur J Pediatr. 2009;168(11):1327-33.

23. Mendonca GV, Pereira FD, Fernhall B. Effects of combined aerobic and resistance exercise training in adults with and without down syndrome. Arch Phys Med Rehabil. 2011;92(1):37-45.

24. Carmeli E, Imam B, Bachar A, Merrick J. Inflammation and oxidative stress as biomarkers of premature aging in persons with intellectual disability. Res Dev Disabil. 2012;33(2):369-75.

25. Davis WE, Sinning WE. Muscle stiffness in Down syndrome and other mentally handicapped subjects: a research note. J Mot Behav. 1987; 19(1):130-44.

26. Shields N, Taylor N, Dodd K. Effects of a community-based progressive resistance training program on muscle performance and physical function in adults with down syndrome: a randomized controlled trial. Arch Phys Med Rehabil. 2008;89(7):1215-20.

27. Hilgenkamp TI, van Wijck R, Evenhuis HM. Feasibility of eight physical fitness tests in 1,050 older adults with intellectual disability: results of the healthy ageing with intellectual disabilities study. Intellect Dev Disabil. 2013;51(1):33-47.

28. Singh SJ, Morgan MD, Hardman AE, Rowe C, Bardsley PA. Comparison of oxygen uptake during a conventional treadmill test and the shuttle walking test in chronic airflow limitation. Eur Respir J. 1994;7 (11):2016-20.

29. Tarantola J, Nardone A, Tacchini E, Schieppati M. Human stance stability improves with the repetition of the task: effect of foot position and visual condition. Neurosci Lett. 1997;228(2):75-8.

30. American College of Sports Medicine. ACSM's guidelines of exercise 
testing and prescription. 10th ed. Alphen aan den Rijn, Netherlands:

Wolters Kluwer Inc. 2018.

31. Rimmer J, Heller T, Wang E, Valerio I. Improvements in physical fitness in adults with down syndrome. Am J Ment Retard. 2004;109(2): $165-74$.

32. Brouwer BJ, Walker C, Rydahl SJ, Culham EG. Reducing fear of falling in seniors through education and activity programs: a randomized trial. J Am Geriatr Soc. 2003;51(6):829-34.

33. Fjeldstad C, Fjeldstad AS, Acree LS, Nickel KJ, Gardner AW. The influence of obesity on falls and quality of life. Dyn Med. 2008;7(1):1-6.

34. Menegoni F, Galli M, Tacchini E, Vismara L, Cavigioli M, Capodaglio P. Gender-specific effect of obesity on balance. Obesity. 2009;17(10):19516.

35. Maffiuletti NA, Agosti F, Proietti M, Riva D, Resnik M, et al. Postural instability of extremely obese individuals improves after a body weight reduction program entailing specific balance training. J Endocrinol
Invest. 2005;28(3):2-7.

36. Teasdale N, Hue O, Marcotte J, Berrigan F, Simoneau M. Reducing weight increases postural stability in obese and morbid obese men. Int J Obes. 2007;31(1):153-60.

37. Cimolin V, Galli M, Grugni G, Vismara L, Precilios H, et al. Postural strategies in prader-willi and down syndrome patients. Res Dev Disabil. 2011;32(2):669-73.

38. Capodaglio P, Menegoni F, Vismara L, Cimolin V, Grugni G, Galli M. Characterisation of balance capacity in prader-willi patients. Res Dev Disabil. 2011;32(1):81-6.

39. Alvarez RG, Marini A, Schmitt C, Saltzman CL. Stage I and II posterior tibial tendon dysfunction treated by a structured nonoperative management protocol: an orthosis and exercise program. Foot Ankle Int. 2006;27(1):2-8.

40. Weber R, French R. Down's syndrome adolescents and strength training. Clin Kinesiol. 1988;42:13-21. 